\title{
A PROPERTY OF POLYNOMIAL EXTENSIONS OF RINGS
}

\section{MICHIO YOSHIDA}

Let $A$ be a commutative ring with unit element, and $A[x]$ $=A\left[x_{1}, \cdots, x_{n}\right]$ be a ring of polynomials in indeterminates $x_{1}, \cdots, x_{n}$ with coefficients in $A$. Let $\mathfrak{a}, \mathfrak{b}, \mathfrak{p}, \mathfrak{q}$ be ideals in $A$, and $\phi(x)$ be a polynomial in $A[x]$. The following facts are quite well known except the last one (vii).

(i) $\phi(x) \in \mathfrak{a} \cdot A[x] \rightleftarrows$ all the coefficients of $\phi(x)$ are in $a$.

(ii) $\mathfrak{a} \cdot A[x] \cap A=\mathfrak{a}$.

(iii) $(\mathfrak{a} \cap \mathfrak{b}) A[x]=\mathfrak{a} \cdot A[x] \cap \mathfrak{b} \cdot A[x]$.

(iv) If $\mathfrak{q}$ is primary with associated prime $\mathfrak{p}$, then $\mathfrak{q} \cdot A[x]$ is primary with $\mathfrak{p} \cdot A[x]$ as its prime $[1$, Lemma 14, p. 85$]$.

(v) If $\phi(x)$ is a zero-divisor in $A[x]$, then there exists an element $c \neq 0$ in $A$ such that $c \phi(x)=0$.

Moreover if $A$ is Noetherian, so is $A[x]$, and

(vi) If $\mathfrak{p}$ is prime, then $\mathfrak{p}$ and $\mathfrak{p} \cdot A[x]$ have the same rank [2, Prop. 5, p. 67].

(vii) If $q$ is primary, then $q$ and $q \cdot A[x]$ have the same length.

We first give a proof of the last assertion (vii), which seems to be less familiar. By passing to the residue ring $A[x] / \mathfrak{q} \cdot A[x]$, we may assume $q=(0)$. Then, let $p$ be the radical of $A$, and $S$ be the complement of $\mathfrak{p}$ in $A$. By forming quotient rings of $A$ and $A[x]$ with respect to $S$, we may assume $A$ is a primary ring with $\mathfrak{p}$ as its unique prime ideal. Let $A \supset \mathfrak{p}=q_{1} \supset q_{2} \supset \cdots \supset q_{l}=(0)$ be a composition series of ideals of $A$. Take arbitrary elements $a_{i}(i=1,2, \cdots, l-1)$ such that $a_{i} \in \mathfrak{q}_{i}, a_{i} \notin \mathfrak{q}_{i+1}$; then $\mathfrak{q}_{i}=\left(\mathfrak{q}_{i+1}, a_{i}\right)$. Suppose $a_{i} \mathfrak{p} \Phi \mathfrak{q}_{i+1}$, then $a_{i} b \notin \mathfrak{q}_{i+1}$ for some element $b$ in $\mathfrak{p}$. Then $a_{i} \in \mathfrak{q}_{i}=\left(\mathfrak{q}_{i+1}, a_{i} b\right)$, hence $a_{i} \equiv a_{i} b c\left(\mathfrak{q}_{i+1}\right)$ for some element $c$ in $A$; since $1-b c$ is a unit, we have $a_{i} \in q_{i+1}$, this is a contradiction. Thus we have $p q_{i} \subseteq q_{i+1}$.

Consider the series: $\mathfrak{p} \cdot A[x] \supset \mathfrak{q}_{2} \cdot A[x] \supset \cdots \supset \mathfrak{q}_{2} \cdot A[x]=(0)$, which we shall prove to be a composition series of the primary ideal (0). For that purpose, let $q^{*}$ be any primary ideal such that $q_{i} \cdot A[x]$ $\supseteq q^{*} \supset q_{i+1} \cdot A[x]$ and take an element $f(x)$ such that $f(x) \in q^{*}$, $f(x) \notin \mathfrak{q}_{i+1} \cdot A[x]$. Then we have $f(x)=g(x)+a_{i} \phi(x)$, with $g(x)$ $\in q_{i+1} A[x] ;$ hence $a_{i} \phi(x) \in q^{*}$ and $a_{i} \phi(x) \notin q_{i+1} \cdot A[x]$. Now we assert $\phi(x) \notin \mathfrak{p} \cdot A[x]$; suppose the contrary: $\phi(x) \in \mathfrak{p} \cdot A[x]$, then $a_{i} \phi(x) \in \mathfrak{q}_{i}$ $\cdot \mathfrak{p} \cdot A[x] \subseteq \mathfrak{q}_{i+1} \cdot A[x]$, a contradiction. Thus, from $a_{i} \phi(x) \in \mathfrak{q}^{*}$ and $\phi(x) \in_{z} \cdot A[x]$ follows $a_{i} \in q^{*}$, whence $q^{*}=q_{i} \cdot A[x]$. This completes the proof.

Received by the editors July 21, 1955 and, in revised form, May 21, 1956. 
From (vi) and (vii) and elementary properties of quotient rings follows immediately the fact:

Let $A=K\left[x_{1}, \cdots, x_{n}\right]$ be a polynomial ring over a field $K$, and let $\mathfrak{p}$ be a prime ideal, $\mathfrak{q}$ be a primary ideal of $A$. Let $L=K(u)$ $=K\left(u_{1}, \cdots, u_{m}\right)$ be a purely transcendental extension of $K$, and form the polynomial ring over $L: R^{*}=L\left[x_{1}, \cdots, x_{n}\right]$. Then $\mathfrak{p} \cdot R^{*}$, $\mathfrak{q} \cdot R^{*}$ are prime and primary respectively. Moreover we have $\operatorname{dim} \mathfrak{p}$ $=\operatorname{dim} \mathfrak{p} \cdot R^{*}$ and length $\mathfrak{q}=$ length $\mathfrak{q} R^{*}$.

This is a special case of a well known theorem. To see this, we have only to consider the intermediary ring: $R_{1}=K[u]\left[x_{1}, \cdots, x_{n}\right]$ $=R[u]$. This is a polynomial extension of $R$, and $R^{*}$ is a quotient ring of $R_{1}$.

Finally we shall note another application of (vii). Let $Q$ be a local ring, $\mathfrak{m}$ be its maximal ideal and put $Q / \mathfrak{m}=k$. Form a polynomial extension of $Q: Q[x]$; and form a quotient ring of $Q[x]$ with respect to its prime ideal $\mathfrak{m} \cdot Q[x]$, which we shall denote by $Q^{*}$. Let $q$ be an $\mathfrak{m}$-primary ideal of $Q$, and $\mathfrak{m}^{*}, \mathfrak{q}^{*}$ be the extensions of $\mathfrak{m}, \mathfrak{q}$ in $Q^{*}$ respectively. Then from (vii) follows that $q$ and $q^{*}$ have the same characteristic function, i.e. $P_{\mathrm{q}}(s)=P_{\mathrm{q}^{*}}(s)$ in Samuel's notation [4]. A fortiori, $\mathfrak{q}$ and $\mathfrak{q}^{*}$ have the same multiplicity: $e(\mathfrak{q})=e\left(\mathfrak{q}^{*}\right)$. We notice the residue field of $Q^{*}$ is $k(x)$. As is well known, a local ring with a finite residue field is sometimes harder to be dealt with. But the above observation suggests that as long as we concern the multiplicities of $m$-primary ideals, there may be some cases where we can overcome the difficulty by passing from $Q$ to $Q^{*}$. We shall illustrate this by quoting a concrete example. Let $l$ be the dimension of $Q$, and assume $l>0$. Let $\mathfrak{n}_{1}, \cdots, \mathfrak{n}_{r}$ be all the $l$-dimensional primary components of the zero ideal of $Q$. Under the hypothesis that $Q$ and its residue field have the same characteristic, D. G. Northcott and D. Rees [3] proved the following interesting fact:

$$
e(\mathfrak{q})=\sum_{i=1}^{r} e\left(\mathfrak{q}+\mathfrak{n}_{i} / \mathfrak{n}_{i}\right) .
$$

Notice that $Q^{*}$ has the same dimension as $Q$, the $l$-dim. primary components of the zero ideal of $Q^{*}$ are $\mathfrak{n}_{1}^{*}, \cdots, \mathfrak{n}_{r}^{*}$, where $\mathfrak{n}_{i}^{*}=\mathfrak{n}_{i} \cdot Q^{*}$, and $e\left(\mathfrak{q}+\mathfrak{n}_{i} / \mathfrak{n}_{i}\right)=e\left(\mathfrak{q}^{*}+\mathfrak{n}_{i}^{*} / \mathfrak{n}_{i}^{*}\right)$. Thus, in proving the above fact, we may assume that $Q$ has an infinite residue field; this remark may bring about a considerable simplification to the proof.

\section{REFERENCES}

1. I. S. Cohen, On the structure and ideal theory of complete local rings, Trans. Amer. Math. Soc. vol. 59 (1946) pp. 54-106. 
2. D. G. Northcott, Ideal theory, Cambridge Univ. Press, 1953.

3. D. G. Northcott and D. Rees, $A$ note on reductions of ideals with application to the generalized Hilbert function, Proc. Cambridge Philos. Soc. vol. 50 (1954) pp. 353359. 1953.

4. P. Samuel, Algèbre locale, Mémorial des Sciences Mathématiques no. 123, Paris,

Hiroshima UNIVERSITY

\title{
CONCERNING REAL VALUED MAPS OF THE $n$-SPHERE ${ }^{1}$
}

\author{
G. R. LIVESAY
}

A definition for the width of a closed curve, believed to be new, is used together with some standard homology theory, to prove the following theorem: Let $f: S^{n} \rightarrow E^{1}$ be continuous, $0 \leqq d \leqq 2, p$ the covering map $S^{n} \rightarrow P^{n}$. If $X_{d}=\left\{x \in S^{n} \mid\right.$ there exists $y \in S^{n}$ with $\rho(x, y)=d, f(x)$ $=f(y)\}$, then $p X_{d}$ carries the nontrivial mod 2 Cech $n-1$ cycle of $P^{n}$. This generalizes Theorem 2 of [4], already considerably extended in other directions by Bourgin [2] and Yang [5].

We will use the following notation: $E^{n+1}=$ Euclidean $n+1$ space, $\rho$ is the Euclidean metric, $\omega$ is the origin in $E^{n+1} . S^{n}=\left\{x \in E^{n+1} \mid \rho(x, \omega)\right.$ $=1\} . P^{n}$ is projective $n$ space. $p_{i}: A_{1} \times A_{2} \rightarrow A_{i}, i=1,2$ will denote the projection. $\mathfrak{K}_{p}(A)=p$ dimensional Cech homology group of $A$ with coefficients the integers mod 2 (the only coefficients to be used here). $T^{2}=S^{1} \times S^{1}, \Delta=$ diagonal in $T^{2}$. In a space $X, \tilde{A}=X-A, \bar{A}$ = closure of $A$.

Lemma 1. Let $g: S^{1} \rightarrow E^{1}$ be continuous, $X$ a connected subset of $T^{2}$ such that either $p_{1} X=S^{1}$ or $p_{2} X=S^{1}$. Then there exists $(x, y) \in X$ such that $g(x)=g(y)$.

Proof. If $p_{1} X=S^{1}$, then there exists $\left(x_{1}, y_{1}\right) \in X$ such that $g\left(x_{1}\right)$ $=\max _{x \in S^{1}} g(x)$, and $\left(x_{2}, y_{2}\right) \in X$ such that $g\left(x_{2}\right)=\min _{x \in S^{1}} g(x)$. Then if neither $\left(g\left(x_{1}\right), g\left(y_{1}\right)\right)$ nor $\left(g\left(x_{2}\right), g\left(y_{2}\right)\right)$ is on the diagonal in $E^{2}$, then they are on opposite sides. If $p_{1} X \neq S^{1}$, then we may make the above argument on the second coordinate.

Presented to the Society December 27, 1956 under the title Concerning real valued maps of $S_{n}$; received by the editors January $12,1957$.

1 The author is grateful to the Office of Naval Research for their support of this research. 